\title{
Acute Mucosal Reactions in Conventional and Concomitant Boost Radiotherapy Schedules in Locally Advanced Oropharayngeal Carcinoma.
}

\author{
Murali Paramanandhan ${ }^{1}$, Shankar Lal Jakhar ${ }^{2}$, Rajesh Kumar ${ }^{3}$, Ramesh Purohit ${ }^{4}$
}

\begin{abstract}
Background: head and neck carcinoma is most common cancer in India and chemo-radiotherapy is the treatment of choice. However altered fractionation schedules have been tried with varying results. The most commonly encountered toxicities during treatment is the mucositis. Aim: compare the mucosal reaction in two radiotherapy schedules in locally advanced oropharyngeal carcinomas. Material and methods: 50 biopsy proven patients with locally advanced oropharyngeal carcinoma were randomised and treated with conventional chemo radiotherapy of $66 \mathrm{~Gy} / 33$ \# with cisplatin $40 \mathrm{mg} / \mathrm{m}^{2}$ weekly. In the other arm, a dose of $67.5 \mathrm{~Gy} / 40 \#$. Results: Grade III mucositis was $32 \%$ vs $24 \%$ (>0.05) in conventional arm and in boost arm respectively. Conclusion: The mucositis was slightly higher in the conventional arm when compared to the boost arm, but was statistically insignificant.
\end{abstract}

Keywords: Radiotherapy, Conventional Fractionation, Altered Fractionation, Mucositis

\section{Introduction}

Head and neck carcinoma is the most common cancer in India with more than $70 \%$ of the cases presenting in advanced stages. $^{[1]}$ The disease remains confined to the loco-regional site of origin in a significant proportion of patients and the most important cause of death is locoregional recurrence. Various studies have shown concurrent chemoradiation schedules to have better locoregional control (LRC) and improved the overall survival. Accelerated fractionation schedules have been studied effectively in head and neck cancers. It is one method which tends to concentrate on shortening the overall treatment time and thus hampering tumor cell proliferation. ${ }^{[2]}$ Concomitant boost is one of the ways of acceleration wherein a second daily fraction is introduced during any phase of treatment, thus completing the treatment in 5-6 weeks. Mucositis and skin reactions are the most commonly encountered acute toxicity during the course of radiotherapy. This is found to be significantly higher in altered fractionation schedules. ${ }^{[3]}$

\section{Aim}

To compare the acute mucosal toxicities in locally advanced (stage III/IV) oro-pharyngeal carcinoma in two different radiotherapy schedules mentioned below.

\section{Material and Methods}

Fifty previously untreated patients of oro-pharyngeal cancer (which include cancer of base of tongue, vallecula and lingual surface of epiglottis, tonsillar region, soft palate and uvula, and oropharyngeal walls), attending the Department of Radiotherapy, Regional Cancer Centre, Bikaner from January 2014 to December 2015 were included in the study fulfilling the following criteria: Biopsy proven cases of squamous cell carcinoma of oropharynx, Stage III, IV, ECOG status 0-1, N-status- upto N2, no co-morbidities. Age- $>18-<70$ years. The evaluation consisted of full medical history, physical examination, local examination and endoscopic assessment of site, nature and extent of the disease was done. Work-up consisted of complete hemogram, renal function test, random blood sugar, X ray chest PA view and $\mathrm{X}$ ray soft tissue neck ( lateral view) and CT scan.

\section{Treatment Planning and Delivery}

All the patients will be treated in a supine position and properly immobilized by the thermoplastic cast (ORFIT cast).

- Dose prescription and Treatment description:

ARM A: The patients were treated by parallel opposing fields (bilateral) and the dose was prescribed to the mid plane at the central axis. Patient in the control arm (CRT arm) received a tumor dose of 66 Gy in 33 fraction over a period of $6 \frac{1}{2}$ weeks, single fraction a day, 5 days a week with concurrent Cisplatin $\left(40 \mathrm{mg} / \mathrm{m}^{2}\right)$ given on weekly. After $44 \mathrm{~Gy} / 22$ fractions and then off cord treatment was continued up to $66 \mathrm{~Gy} / 33$ fractions.

-ARM B: $45 \mathrm{~Gy} / 25$ fractions/5weeks, $1.8 \mathrm{~Gy} /$ fraction/day, 5days/week was delivered to the large field comprising the primary disease with extension and the neck nodes. Simultaneously a boost of $22.5 \mathrm{~Gy} / 15$ fractions, $1.5 \mathrm{~Gy} /$ fraction on alternate day from the $1^{\text {st }}$ day of the treatment for 5 weeks to the primary disease. The fractions were delivered 6 hours apart.

- All patients were planned and treatment was delivered on Cobalt-60 Bhabhatron. Off cord planning was done after delivering 45 Gy.

- The patients in both arms were assessed after every 5 fraction for treatment related acute toxicity.

- The treatment was stopped when the patient developed grade 4 mucosal reactions.

- The Biologically Effective Doses (BED) for Tumor control and late adverse effects in each arm will be calculated using the linear quadratic equation. BEDs for both arm is as follows:

-ARM A: 79.2 Gy for Tumor Control and 110 Gy for late reacting normal tissues complications.

-ARM B: 78.98 Gy for Tumor control and 105.75 Gy for late reacting normal tissue complication. 


\section{International Journal of Science and Research (IJSR) \\ ISSN (Online): 2319-7064}

Index Copernicus Value (2013): 6.14 | Impact Factor (2015): 6.391

Acute treatment related toxicity assessed and graded using common terminology criteria for adverse events (RTOG). The two arms were compared statistically and $p$ values were calculated using online.

\section{Results}

Male: female- 5:1, Age- $<50$ years-23, $>50$ years- 27, stage III- 28, stage IV- 22. Grade III mucositis was $32 \%$ vs $24 \%$ $(>0.05)$ in conventional arm and in boost arm respectively.

\section{Discussion}

The treatment of loco regionally advanced head and neck cancers has undergone a paradigm shift over the past three decades, with management strategies changing from surgery or radiation therapy as single modality to combined modality treatment. A pragmatic approach is to evaluate alternative and viable radiation schedules that provide superior response rates than conventional radiation and yet maintain a favourable toxicity profile, unlike the chemo radiation regimens.

Concomitant boost radiotherapy technique has a strong radiobiological rationale, wherein the boost field is added to the ongoing radiotherapy portals during any phase of radiation to counteract accelerated repopulation of the tumor clonogens. ${ }^{[3,4]}$ This basic premise enhances the chances of tumor control by using a larger dose per fraction in the area of gross tumor volume and at the same time maintains the beneficial effects of acceleration on the tumor and normal tissues. This seemed important not only to optimize the therapeutic ratio, but also the available resources, as this schedule leads to an abbreviation of the overall treatment time from 7 to 5 weeks.

Incidence of disease is more common in male with male: female ratio of $5: 1$. As per the literature the male to female ratio of head and neck cancers varies from $3-5: 1 .^{[5]}$ Mucositis is a major complication of any form of radiation therapy in head and neck cancers. It has a major impact on the overall quality of life during treatment, as well as after treatment. Trotti et al have reviewed the effect of mucositis on patients with head and neck cancers using conventional radiotherapy, accelerated radiotherapy [RT-AF] and chemoradiation $[\mathrm{RT}+\mathrm{CT}]$. The frequency of mucositis was highest in patients with RT-AF, affecting $100 \%$ of patients overall. Patients treated with RT-AF experienced the most severe mucositis, with more than half $(57 \%)$ experiencing grade 3-4 mucositis. ${ }^{[6]}$ Alteration of fractionation scheme has mucositis rate of $60 \%$ in various studies. ${ }^{[7]}$ In this study, grade III mucositis was encountered in $32 \%$ of patients in arm A compared to $24 \%$ in arm B $(P>0.05)$. This could be explained by alternate day boost gives enough time for normal tissue recovery whereas the mucositis with conventional arm in higher due to chemotherapy given weekly as well as longer duration of treatment. The patients in both arms were prophylactically maintained on oral gargles in form of betadine and soda bicarbonate. Also, symptomatic treatment was given whenever necessary.

\section{Conclusion}

The mucositis was slightly higher in the conventional arm when compared to the boost arm, but was statistically insignificant.

\section{References}

[1] National Cancer Registry Programme: Consolidated Report of Hospital Based Registries, 1994-1998.

[2] Overgaard J, Hansden SH, Sapru W. Conventional radiotherapy as the primary treatment of squamous cell carcinoma of the head and neck. A randomized multicenter study of 5 versus 6 fractions per weekpreliminary report from the DAHANCA 6 and 7 trial. Radiother Oncol 1996;40:S31 .

[3] Sasaki T, Sato Y, Sakka M. Cell population kinetics of human solid tumors: a statistical analysis in various histological types. Gann. 1980;71(4):520-9.

[4] Thames HD, Jr., Peters LJ, Withers HR, Fletcher GH. Accelerated fractionation vs hyperfractionation: rationales for several treatments per day. Int $\mathrm{J}$ Radiat Oncol Biol Phys. 1983;9(2):127-38.

[5] Bjordal K, Ahlner-Elmqvist M, Tollesson E, Jensen AB, Razavi D, Maher EJ, et al. Development of a European Organization for Research and Treatment of Cancer (EORTC) questionnaire module to be used in quality of life assessments in head and neck cancer patients. EORTC Quality of Life Study Group. Acta Oncol. 1994;33(8):879-85.

[6] Trotti A, Colevas AD, Setser A, Rusch V, Jaques D, Budach V, et al. CTCAE v3.0: development of a comprehensive grading system for the adverse effects of cancer treatment. Semin Radiat Oncol. 2003;13(3):17681.

[7] Valentina Krstevska and Simonida Crvenkova Altered and Conventional Fractionated Radiotherapy in Locoregional Control and Survival of Patients with Squamous Cell Carcinoma of the Larynx, Oropharynx, and Hypopharynx. Croat Med J. 2006; 47(1): 42-52. 